\title{
Clinical pharmacology aspects in patients treated with TNF inhibitors during SARS-Cov-2 pandemic
}

\author{
Antonio Vitiello ${ }^{1}$, Chiara Pelliccia ${ }^{2}$, Francesco Ferrara ${ }^{1}$, and Raffaele La Porta ${ }^{3}$ \\ ${ }^{1}$ Azienda Unità Sanitaria Locale Umbria 1 \\ ${ }^{2}$ Azienda Unità Sanitaria Locale Umbria 2 \\ ${ }^{3}$ ASUR Marche
}

April 29, 2020

\begin{abstract}
In this period of global pandemic caused by SARS-Cov-2, it is of paramount importance to recognize all risk factors that may increase the likelihood of infection. In addition to the risk factors known as pre-existing diseases and old age, risk factors could be drug treatments for chronic diseases, such as immunomodulating drugs that can alter immune defences and response to infectious agents. Antibodies that inhibit tumor necrosis factor (TNF) such as adalimumab infliximab etanercept and golimumab have been used for over 20 years in severe cases of autoimmune diseases such as rheumatoid arthritis, inflammatory bowel disease or ankylosing spondylitis. Due to their mechanism of action they reduce inflammation and can stop the progression of the disease by inhibiting a key factor of inflammation such as Tumor Necrosis Factor (TNF). In this article we want to examine the possible correlation between therapy with TNF inhibitors and the increased risk of SARS-CoV-2 infection, and the possible paradoxical therapeutic efficacy in patients with ongoing infection, especially in phase two and three. We express our opinion on this very complex and sensitive topic which is the subject of discussion among physicians and experts, based on current knowledge of the literature. Keywords: TNF inhibitors, SARS-Cov-2, immunomodulating, infection, hyperinflammatory
\end{abstract}

\section{Hosted file}

TNF inhibitors during SARS-Cov-2 pandemic.rtf available at https://authorea.com/users/310215/ articles/446693-clinical-pharmacology-aspects-in-patients-treated-with-tnf-inhibitors-duringsars-cov-2-pandemic 